\title{
High-pressure Helium as a Template for the Formation of a Microporous Silica Structure
}

\author{
Sergey V. Kukhtetskiy*, \\ Elena V. Fomenko and Elena S. Rogovenko \\ Institute of Chemistry and Chemical Technology SB RAS \\ Federal Research Center "Krasnoyarsk Scientific Center of the SB RAS» \\ Krasnoyarsk, Russian Federation
}

Received 13.07.2021, received in revised form 01.02.2022, accepted 04.02.2022

\begin{abstract}
The modeling of various modes of formation of microporous silica using high-pressure helium was carried out by the methods of molecular dynamics. It is shown that by controlling the helium pressure in the process of quenching the silica melt, the structure of the obtained glasses can be changed continuously and within wide limits. The density of the obtained glasses varies in the range from 2.2 to $1.5 \mathrm{~g} / \mathrm{cm}^{3}$ and the Ostwald solubility coefficients for helium and neon are more than an order of magnitude.
\end{abstract}

Keywords: helium, neon, silica, expanded quartz glass, glass transition temperature, solubility.

Acknowledgements. This work was conducted within the framework of the budget project \# 02872021-0013 for Institute of Chemistry and Chemical Technology SB RAS.

Citation: Kukhtetskiy, S. V., Fomenko, E. V., Rogovenko, E. S. High-pressure helium as a template for the formation of a microporous silica structure. J. Sib. Fed. Univ. Chem., 2022, 15(1), 118-127. DOI: 10.17516/1998-2836-0278

(C) Siberian Federal University. All rights reserved

This work is licensed under a Creative Commons Attribution-Non Commercial 4.0 International License (CC BY-NC 4.0).

* Corresponding author E-mail address: kswrk@mail.ru 


\title{
Гелий высокого давления как темплат
}

\section{Для формирования \\ микропористой структуры кремнезема}

\author{
С. В. Кухтецкий, Е. В. Фоменко, Е. С. Роговенко \\ Институт химии и химической технологии СО РАН \\ ФИЦ «Красноярский научный изентр СО РАН» \\ Российская Федераџия, Красноярск
}

\begin{abstract}
Аннотация. Методами молекулярной динамики проведено моделирование различных режимов формирования микропористого кремнезема при помощи гелия высокого давления. Показано, что путем регулирования давления гелия в процессе закалки расплава кремнезема можно непрерывно и в широких пределах изменять структуру получаемых стекол. Плотность в полученных стеклах изменяется в диапазоне от 2.2 до 1.5 г / см³ , а коэффициенты растворимости Оствальда для гелия и неона - более чем на порядок.
\end{abstract}

Ключевые слова: гелий, неон, кремнезем, расширенное кварцевое стекло, температура стеклования, растворимость.

Благодарности. Исследование выполнено в рамках государственного задания Института химии и химической технологии СО РАН (проект 0287-2021-0013).

Цитирование: Кухтецкий, С. В. Гелий высокого давления как темплат для формирования микропористой структуры кремнезема / С. В. Кухтецкий, Е. В. Фоменко, Е. С. Роговенко // Журн. Сиб. федер. унта. Химия, 2022, 15(1). С. $118-127$. DOI: $10.17516 / 1998-2836-0278$

\section{Введение}

Повышенный интерес к пористым стеклам связан с их многочисленными приложениями в химии, фармацевтике и биологии [1-9]. Благодаря целому ряду уникальных физикохимических свойств, таких как химическая, термическая и механическая стойкость, пористые стекла используются в качестве мембранных материалов для разделения газов $[10,11]$, носителей катализаторов [12-14], инкапсуляции ядерных отходов [15-17], в качестве основы оптических хемосенсоров $[18,19]$, фильтрующих материалов $[20,21]$ и во многих других прикладных задачах. На данный момент существуют две основные технологии получения пористых стекол [1]. Первая основана на кислотном выщелачивании боратной фазы в боросиликатном стекле с разделенными фазами [2-4], вторая - на формировании/добавлении органического темплата в рамках золь-гель процесса с последующим кальцинированием и удалением продуктов распада темплата [5-9]. Обеим технологиям присущи определенные сложности, связанные с регулированием расслоения фаз, размеров пор, топологией свободного пространства и необходимостью удаления боратной подсистемы или темплата, в случае золь-гель процесса. 
В этой связи представляет интерес использование легких инертных газов, в особенности гелия, для модификации микропористой структуры кремнезема. Первая причина такого интереса заключается в очень высокой растворимости гелия высокого давления в кремнеземе. Недавние исследования поведения кварцевого стекла в атмосфере гелия [22, 23] показали аномально низкую сжимаемость стекла по сравнению с аналогичными измерениями в других средах. Верхняя оценка растворимости, сделанная в [22], дает значение 2.4 моля Не на моль $\mathrm{SiO}_{2}$ при давлении $10 \mathrm{GPa}$. Это говорит о том, что гелий заполняет все междоузельное пространство стекла. Вторая причина, обуславливающая перспективность использования гелия, заключается в его высокой проникающей способности, позволяющей проводить эффективную эвакуацию темплата при сравнительно низких $\left(300-400{ }^{\circ} \mathrm{C}\right)$ температурах. Третья очевидная причина - химическая инертность гелия, исключающая загрязнения результирующих стекол углеродом и другими продуктами разложения органических темплатов.

В данной работе описаны результаты численных экспериментов по модификации кварцевого стекла при помощи гелия высокого давления, выполненных методом молекулярной динамики. Представлены структуры модифицированных стекол, зависимости плотности и коэффициентов растворимости от режимов закалки расплава.

\section{Методики расчета \\ и вспомогательные результаты}

\section{Получение образиов расширенного квариевого стекла}

В качестве исходных конфигураций использовались образцы кварцевых стекол, полученные и охарактеризованные в работе [24]. Расчетная ячейка (кубическая с периодическими граничными условиями) содержит 1000 ионов кремния и 2000 ионов кислорода. Начальное распределение скоростей частиц соответствует начальной температуре термостата (300 K). Затем в случайных точках междоузельного пространства размещались атомы гелия. В данной работе количество атомов равно 1000, что соответствует единичному коэффициенту растворимости Оствальда. Распределение атомов гелия по скоростям соответствовало температуре $300 \mathrm{~K}$. При размещении атомов применяли следующее правило: если кинетическая энергия данного атома была не меньше его потенциала в данной точке, то атом добавлялся в систему, если нет, то выбиралась другая случайная точка свободного пространства. Затем устанавливалось нужное давление баростата и запускался расчет динамики. В данных численных экспериментах начальное давление баростата устанавливалось на $8 \mathrm{GPa}$. Динамика рассчитывалась в рамках NPT-ансамбля. Типичные диаграммы регулирования температуры и давления ансамбля показаны на рис. 1.

В течение 0.1 нс производится линейный нагрев системы до температуры гомогенизации. После гомогенизации осуществляется первая стадия закалки (линейное снижение температуры системы при постоянном давлении) до некоторой температуры $\mathrm{T}_{\mathrm{p}}$, при которой производится сброс давления до атмосферного. После чего закалка продолжается до комнатной температуры при нормальном давлении и стадии релаксации стекла в течение последних 0.1 нс. Варьированием температуры сброса давления $\left(\mathrm{T}_{\mathrm{p}}\right)$ достигаются различные степени расширения кварцевого стекла. 

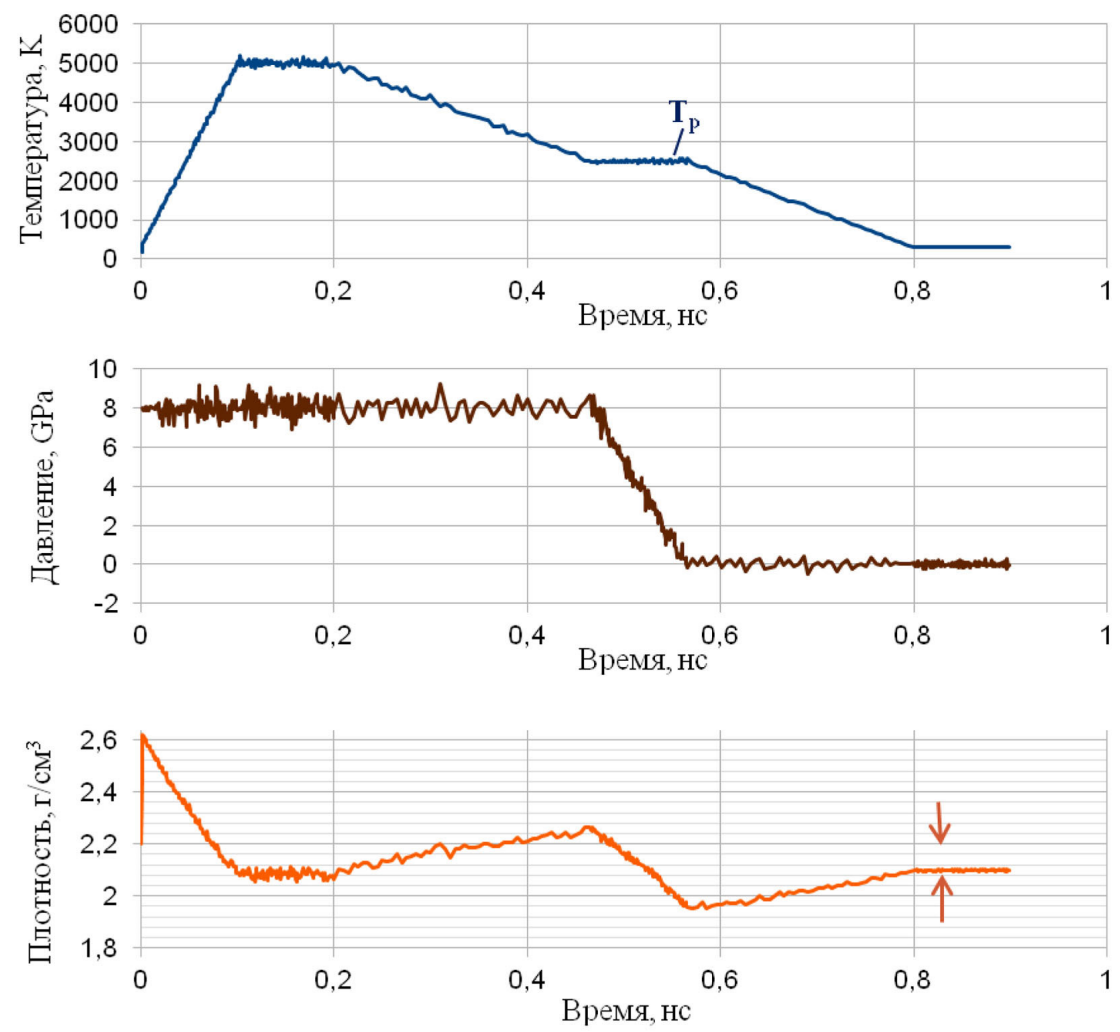

Рис. 1. Типичные диаграммы регулирования температуры и давления NPT-ансамбля $\mathrm{SiO}_{2}+\mathrm{He}$ во время процесса расширения стекла. Начальное состояние системы: $\mathrm{T}=300 \mathrm{~K}, \mathrm{P}=8$ ГПа. Т - температура сброса давления. Конечное состояние: $\mathrm{T}=300 \mathrm{~K}, \mathrm{P}=0.1 \mathrm{MПа.} \mathrm{На} \mathrm{нижней} \mathrm{диаграмме} \mathrm{показана} \mathrm{зависимость}$ плотности матрицы (без учета растворенного гелия) от времени. Стрелками показано изменение плотности стекла относительно исходной плотности кварцевого стекла, равной 2.2 г / см ${ }^{3}$

Fig. 1. Typical diagrams of temperature and pressure control of NPT-ensemble $\mathrm{SiO}_{2}+\mathrm{He}$ during glass expansion process. Initial state of the system: $\mathrm{T}=300 \mathrm{~K}, \mathrm{P}=8 \mathrm{GPa} . \mathrm{T}_{\mathrm{p}}$ is the pressure relief temperature. Final state: $\mathrm{T}=300$ $\mathrm{K}, \mathrm{P}=0.1 \mathrm{MPa}$. The lower diagram shows the dependence of the matrix density (excluding dissolved helium) on time. The arrows show the change in glass density relative to the initial density of quartz glass, equal to $2.2 \mathrm{~g} / \mathrm{cm}^{3}$

\section{Вычисление температуры стеклования}

Температура сброса давления $\mathrm{T}_{\mathrm{p}}$ привязана к температуре стеклования $\mathrm{T}_{\mathrm{g}}$ (температура, при которой происходит резкий рост вязкости расплава при закалке). В литературе отсутствовали данные о температуре стеклования расплава кварца в атмосфере гелия высокого давления. Поэтому было проведено отдельное исследование зависимости $\mathrm{T}_{\mathrm{g}}$ от давления гелия. В процессе закалки при прохождении точки стеклования происходит формирование окончательной структуры стекла. Поэтому наиболее простым способом вычисления $\mathrm{T}_{\mathrm{g}}$ является определение точки излома на зависимости удельной потенциальной энергии системы от температуры. Пример такой диаграммы показан на рис. 2. По пересечению линий линейных регрессий левой и правой ветвей находилась температура стеклования.

Температуру сброса давления в процессе закалки $\mathrm{T}_{\mathrm{p}}$ удобно отсчитывать в единицах температуры стеклования $\mathrm{T}_{\mathrm{g}}$, в соответствии с этим на рис. 3 приведена диаграмма зависимости $\mathrm{T}_{\mathrm{g}}$ от давления, рассчитанная по указанной выше методике. 


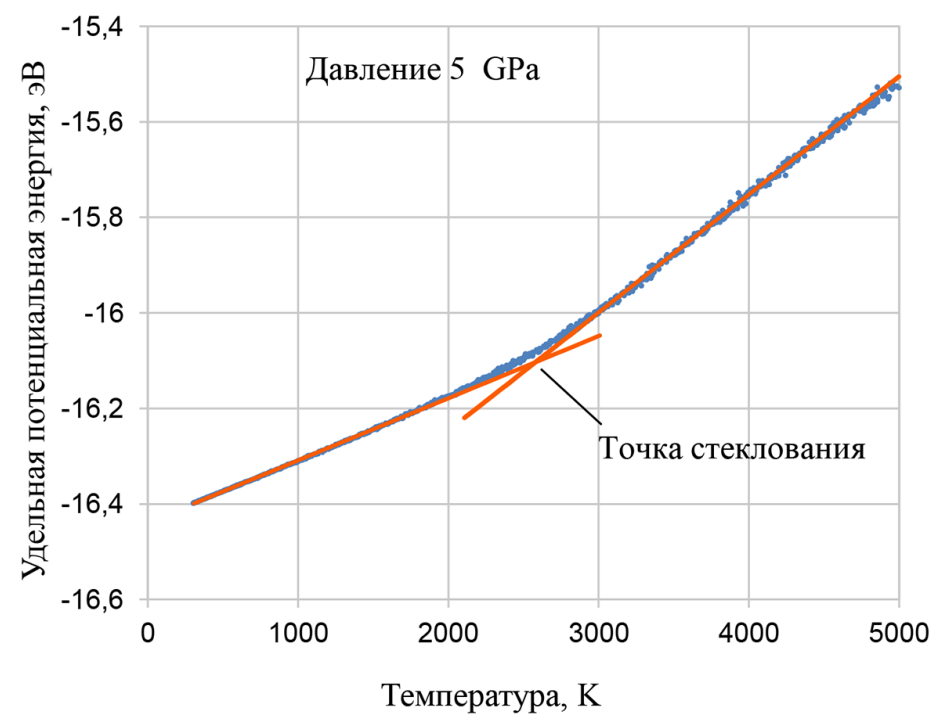

Рис. 2. Зависимость удельной потенциальной энергии системы $\mathrm{SiO}_{2}-$ Не от температуры $(\mathrm{P}=5$ ГПа)

Fig. 2. Dependence of the specific potential energy of the $\mathrm{SiO}_{2}-\mathrm{He}$ system on temperature $(\mathrm{P}=5 \mathrm{GPa})$

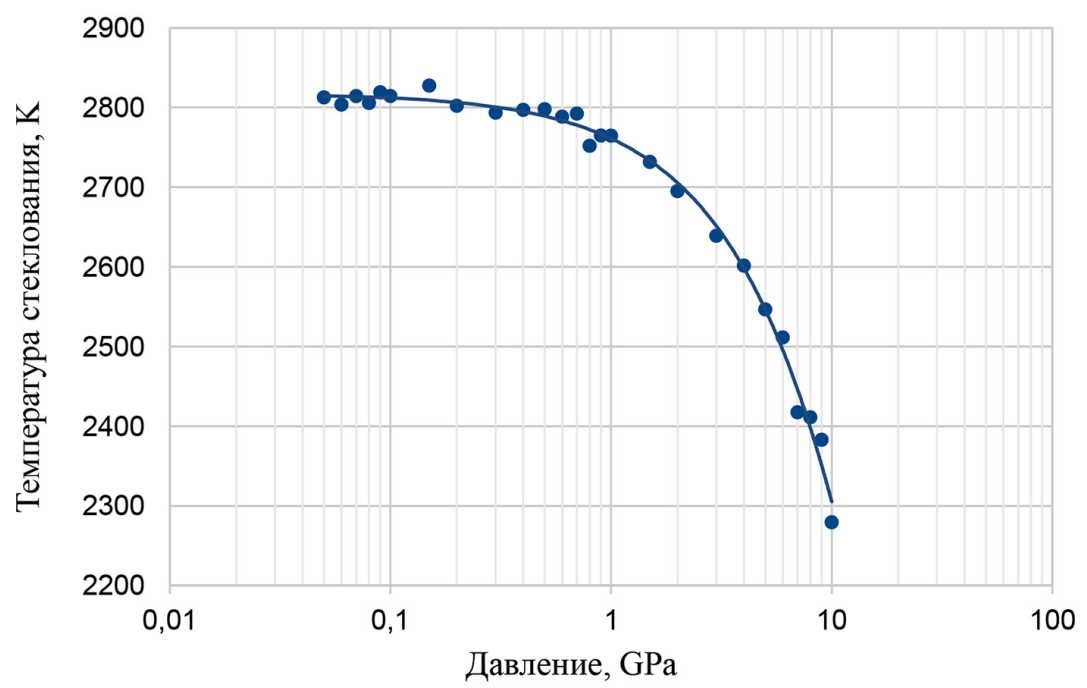

Рис. 3. Зависимость температуры стеклования $\mathrm{T}_{\mathrm{g}}$ от давления гелия

Fig. 3. Dependence of the glass transition temperature $T_{g}$ on the helium pressure

\section{Вычисление растворимости}

Методика вычисления растворимости подробно описана в [25]. Суть ее заключается в построении дискретного 3D-потенциального пробного атома (Не или $\mathrm{Ne}$ ) для каждого образца стекла с последующим суммированием Больцмановского фактора по всем ячейкам (вокселам). Размер воксельного пространства 256х256х256 ячеек. В расчетах использовались потенциалы взаимодействия гелия и неона с матрицей кремнезема, полученные в этой же работе [25]. 


\section{Результаты}

На рис. 4 изображена зависимость плотности расширенного кварцевого стекла в зависимости от температуры сброса давления при закалке. Первоначальное давление системы $8 \mathrm{GPa}$, рассчитанная температура стеклования при этом давлении $\mathrm{T}_{\mathrm{g}}=2400 \mathrm{~K}$. Относительная температура сброса давления $\mathrm{T}_{\mathrm{p}} / \mathrm{T}_{\mathrm{g}}$ изменялась в широком диапазоне - от 0.1 до 1.7. Сверху и снизу диаграммы приведены типичные атомные конфигурации получающихся в результате закалки стекол. Белым цветом изображены границы полостей (пор).

В целом весь диапазон $\mathrm{T}_{\mathrm{p}} / \mathrm{T}_{\mathrm{g}}$ можно разбить на две области.

1. $\mathrm{T}_{\mathrm{p}} / \mathrm{T}_{\mathrm{g}}<1.4$. Это участок однородного расширения структуры расплава. После сброса внешнего давления растворимость гелия заметно падает. Излишки гелия, растворенного в свободном пространстве кремнезема, начинают выделяться из расплава, приводя к локальному разрыхлению молекулярной структуры (рис. 4, две верхних конфигурации). Но точка стеклования и, соответственно, резкий рост вязкости достигаются раньше, чем происходит полное разделение фаз и формирование более крупных пузырьков гелия. В результате такая достаточно однородная «разрыхленная» структура расплава замораживается и остается уже в затвердевшем стекле. Максимальное однородное расширение кварцевого стекла (уменьшение плотности), достигнутое в данных численных экспериментах, составляет приблизительно $10 \%$ при $\mathrm{T}_{\mathrm{p}} / \mathrm{T}_{\mathrm{g}}$ около 1.4 .

2. $1.4<\mathrm{T}_{\mathrm{p}} / \mathrm{T}_{\mathrm{g}}$. Это область образования «нанопены». Фазы гелия и кремнезема успевают частично разделиться до достижения расплавом точки стеклования. Часть гелия образует пузырьки, размер которых зависит от $\mathrm{T}_{\mathrm{p}} / \mathrm{T}_{\mathrm{g}}$, часть остается в свободном пространстве кремнезема, приводя к его частичному расширению. После прохождения точки стеклования образованная структура расплава «замораживается» и формирует структуру среднего порядка уже твердого стекла. Как видно на рис. 4, наличие и размеры пор при заданной скорости закалки тоже можно регулировать посредством изменения $\mathrm{T}_{\mathrm{p}} / \mathrm{T}_{\mathrm{g}}$.

Так как состав кварцевого стекла в рассматриваемых процессах остается неизменным, то степень его расширения, как видно из сравнения диаграммы плотности (в центре рис. 4) и атомных конфигураций (сверху и снизу рис. 4), можно однозначно характеризовать одним параметром - плотностью стекла. Но этот интегральный показатель относительно слабо изменяется при однородном расширении расплава (всего до $10 \%$ при $\mathrm{T}_{\mathrm{p}} / \mathrm{T}_{\mathrm{g}}=1.4$ ). Более чувствительным индикатором изменения топологии свободного пространства является растворимость легких инертных газов, таких как гелий или неон. На рис. 5 показаны зависимости коэффициентов растворимости Оствальда для гелия и неона (при $300 \mathrm{~K}$ ) от относительной температуры сброса давления $\mathrm{T}_{\mathrm{p}} / \mathrm{T}_{\mathrm{g}}$. Для сравнения на этой же диаграмме слева (квадратные точки) представлены коэффициенты растворимости Не и $\mathrm{Ne}$ в обычном (исходном) кварцевом стекле, усредненные по пяти различным образцам.

Из представленных на рис. 5 диаграмм видно, что до $\mathrm{T}_{\mathrm{p}} / \mathrm{T}_{\mathrm{g}}<1$ коэффициенты растворимости практически не изменяются. То есть геометрия свободного пространства будущего стекла, сформированная гелием, растворенным в расплаве, «замораживается» сразу же после прохождения точки стеклования. Вполне естественно, что эта геометрия немного расширена по сравнению со свободным пространством обычного кварцевого стекла за счет растворенного в расплаве гелия.

$$
-123-
$$




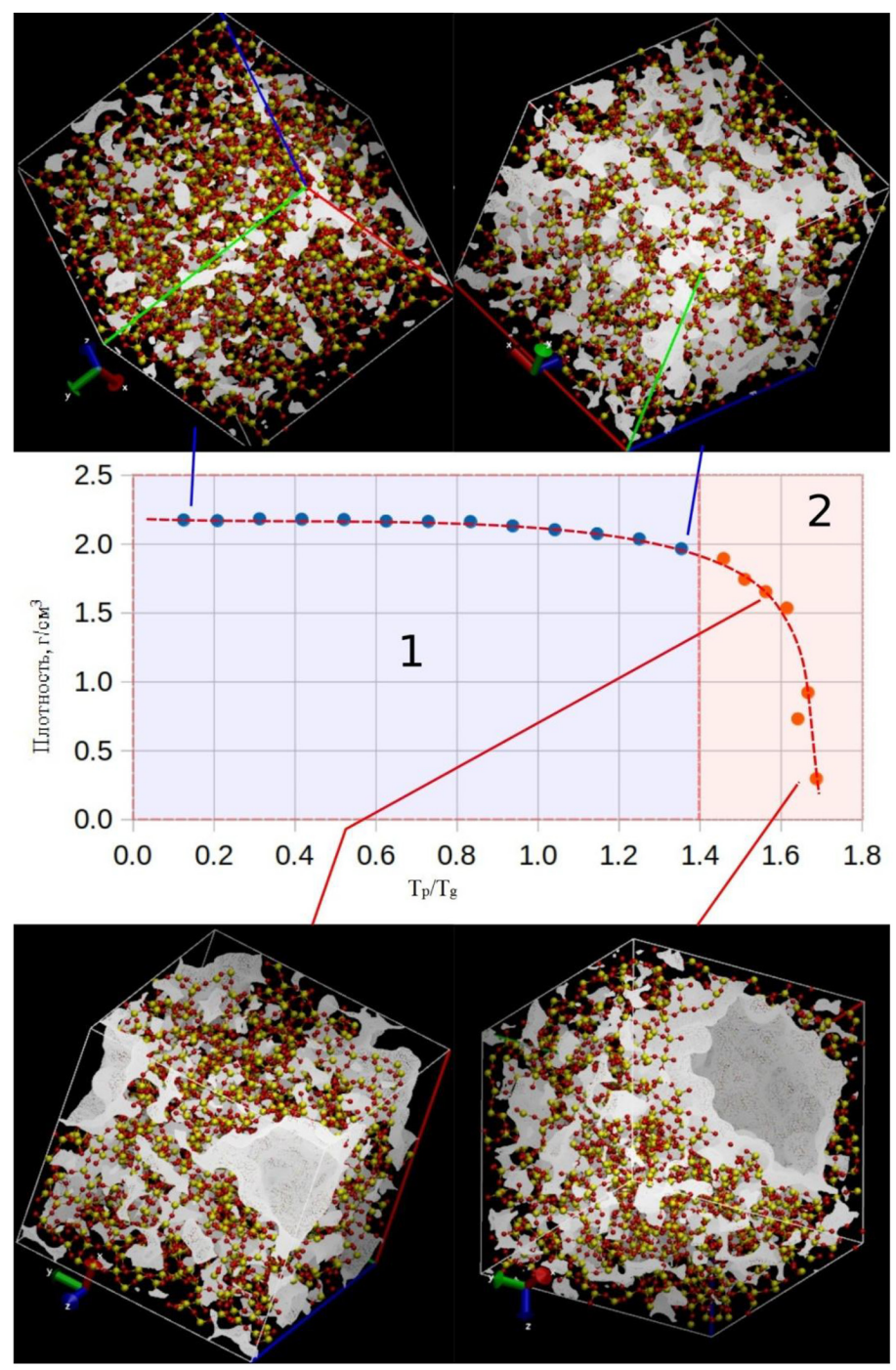

Рис. 4. Зависимость плотности расширенного кварцевого стекла от относительной температуры сброса давления $\mathrm{T}_{\mathrm{p}} / \mathrm{T}_{\mathrm{g}}$. Сверху и снизу - типовые атомные конфигурации результирующих стекол. Белым изображено свободное пространство (полости)

Fig. 4. Dependence of the density of expanded quartz glass on the relative depressurization temperature $\mathrm{T}_{\mathrm{p}} / \mathrm{T}_{\mathrm{g}}$. Above and below - typical atomic configurations of the resulting glasses. Freespace (cavities) is shown in white

При более раннем сбросе давления, выше точки стеклования $\left(\mathrm{T}_{\mathrm{p}} / \mathrm{T}_{\mathrm{g}}>1\right)$, легко видеть быстрый (практически экспоненциальный) рост растворимости с увеличением $\mathrm{T}_{\mathrm{p}} / \mathrm{T}_{\mathrm{g}}$. Такое поведение системы дает возможность регулировать пористость получающихся стекол в достаточно широких пределах. 


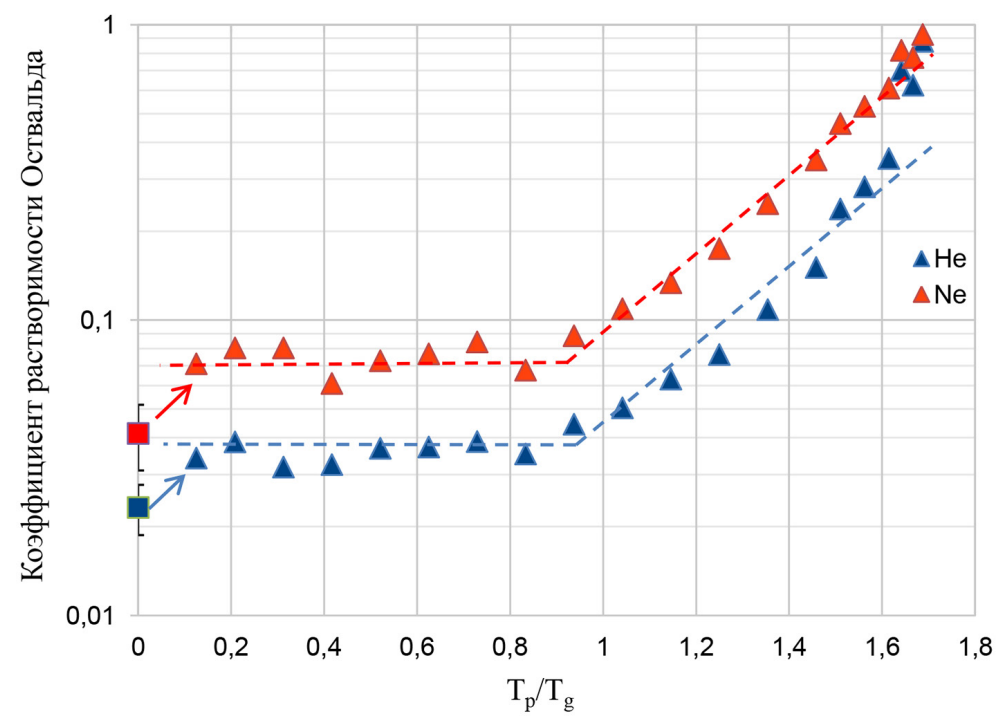

Рис. 5. Зависимости коэффициентов растворимости Оствальда при $300 \mathrm{~K}$ от относительной температуры сброса давления $\mathrm{T}_{\mathrm{p}} / \mathrm{T}_{\mathrm{g}}$. Для сравнения слева (квадратные точки) показаны коэффициенты растворимости Не и $\mathrm{Ne}$ в обычном (исходном) кварцевом стекле, усредненные по пяти различным образцам

Fig. 5. Dependences of the Ostwald solubility coefficients at $300 \mathrm{~K}$ on the relative depressurization temperature $\mathrm{T}_{\mathrm{p}} / \mathrm{T}_{\mathrm{g}}$. For comparison, on the left (square dots) the solubility coefficients of He and $\mathrm{Ne}$ in ordinary (original) quartz glass are shown averaged over 5 different samples

\section{Выводы}

Методами молекулярной динамики проведено моделирование процесса закалки расплава кремнезема в атмосфере гелия высокого давления (до $10 \mathrm{GPa}$ ). Показано, что при сбросе давления в процессе закалки образуются микропористые стекла двух типов, зависящие от относительной температуры сброса давления $\mathrm{T}_{\mathrm{p}} / \mathrm{T}_{\mathrm{g}}$. В первом случае при $\mathrm{T}_{\mathrm{p}} / \mathrm{T}_{\mathrm{g}}<1.4$ образуются однородно расширенные стекла без крупных пор. Во втором - при $\mathrm{T}_{\mathrm{p}} / \mathrm{T}_{\mathrm{g}}>1.4$ фазы гелия и расплава кремнезема частично разделяются, образуя в отвердевшем стекле более крупные поры (порядка нанометра и более), разделенные перегородками, имеющими расширенную структуру по типу 1. Рассчитанные параметры расширенных стекол (плотность и коэффициенты растворимости гелия и неона) показывают монотонную зависимость степени расширения стекла от параметра $\mathrm{T}_{\mathrm{p}} / \mathrm{T}_{\mathrm{g}}$, что дает возможность управлять геометрией свободного пространства стекол.

\section{Список литературы / References}

1. Hasanuzzaman M., Rafferty A., Sajjia M., Olabi A.-G. Production and treatment of porous glass materials for advanced usage. Reference Module in Materials Science and Materials Engineering 2016. P. $1-11$.

2. Patent 2106744 US. Hood H.P., Nordberg M.E. Treated borosilicate glass. Publ. Date 01.02.1938).

3. Tanaka H., Yazawa T., Eguchi K., Nagasawa H., Matsuda N., Einishi T. Precipitation of colloidal silica and pore size distribution in high silica porous glass. Journal of Non-Crystalline Solids 1984. Vol. 65. P. 301-309.

$$
-125-
$$


4. McMillan P.W., Matthews C.E. Microporous glasses for reverse osmosis. Journal of Materials Science 1976. Vol. 11. P. 1187-1199.

5. Raman N.K., Brinker C. J. Organic template approach to molecular sieving silica membranes. Journal of Materials Science 1995. Vol. 105. P. 273-279.

6. Li G., Kanezashi M., Tsuru T. Preparation of organic-inorganic hybrid silica membranes using organoalkoxysilanes: The effect of pendant groups. Journal of Materials Science 2011. Vol. 379, P. 287-295.

7. Cao G.Z., Brinker C.J. Gas permeation and microstructure of silica membranes prepared using an organic template approach by sol-gel processing. Materials Research Society Symposium Proceedings 1996. Vol. 431. P. 343-348.

8. Kamimura Y., Miyoshi K., Kurumada K. Fabrication of microporous amorphous silica glass by pyrolysis of phenyl groups intercalated in sol-gel derived phenyl-modified silica glass. Journal of Non-Crystalline Solids 2010. Vol. 356. P. 1842-1847.

9. Rios X., Moriones P., Echeverria J.C., Luquin A., Laguna M., Garrido J. J. Ethyl group as matrix modifier and inducer of ordered domains in hybrid xerogelssynthesised in acidic media using ethyltriethoxysilane (ETEOS) and tetraethoxysilane (TEOS) as precursors. Materials Chemistry and Physics 2013. Vol. 141. P. 166-174.

10. Enke D., Janowskia F., Schwiegerb W. Porous glasses in the 21st century - a short review. Microporous and Mesoporous Materials 2003. Vol. 60. P. 19-30.

11. Kuraoka K., Hirano T., Yazawa T. High-selectivity, high-flexibility glass hollow-fiber membrane for gas separation. Chemical Communications 2002. P. 664-665.

12. Polshettiwara V., Molnar A. Silica-supported Pd catalysts for Heck coupling reactions. Tetrahedron 2007. Vol. 63. P. 6949-6976.

13. Lee D.-H., Jung J.-Y., Jin M.-J. Highly active and recyclable silica gel-supported palladium catalyst for mild cross-coupling reactions of unactivatedheteroaryl chlorides. Green Chemistry 2010. Vol. 12. P. 2024-2029.

14. Polshettiwara V., Lenb C., Fihri A. Silica-supported palladium: Sustainable catalysts for cross-coupling reactions. Coordination Chemistry Reviews 2009. Vol. 253. P. 2599-2626.

15. Соболев И.А., Ожован М.И., Щербатова Т.Д., Батюхнова О.Г. Стекла для радиоактивных отходов. М.: Энергоатомиздат, 1999. 238 с. [Sobolev I.A., Ozhovan M.I., Shcherbatova T. D., Batyuhnova O. G. Glasses for radioactive waste. Moscow: Energoatomizdat, 1999. 238 p. (In Russ.)]

16. Вашман А.А., Демин А.В., Крылова Н.В., Кушников В.В., Матюнин Ю. И., Полуэктов П.П., Поляков А.С., Тетерин Э.Г. Фосфатные стекла с радиоактивными отходами. М.: ЦНИИатоминформ, 1997. 172 с. [Vashman A.A., Demin A.V., Krilova N.V., Kyshnikov V.V., Matyunin Yu.I., Polyektov P.P., Polyakov A.S., Teterin E. G. Phosphate glasses with radioactive waste. Moscow: CNIIatominform, 1997. 172 p. (In Russ.)]

17. Jantzen C.M., Kaplan D. I., Bibler N. E., Peeler D. K., Plodinec M. J. Performance of a buried radioactive high level waste (HLW) glass after 24 years. Journal of Nuclear Materials 2008. Vol. 378. P. 244-256.

18. Evstrapov A. Esikova N., Rudnitskaya G., Antropova T.V. Porous glasses as a substrate for sensor elements. Optica Applicata 2010. Vol. 4 (2). P. 333-340.

$$
-126-
$$


19. Memisevic J., Korampally V., Gangopadhyay S., Grant S. A. Characterization of a novel ultra-low refractive indexmaterial for biosensor application. Sensors and Actuators B Chemical 2009. Vol. 141. P. 227-232.

20. Ермакова Л.Э., Сидорова М.П., Цыганкова Т.А. Структурные параметры и обменная емкость мембран из пористых стекол. Коллоидный журнал 1990. № 4. С. 774-777. [Ermakova L.E., Sidorova M.P., Tsygankova T. V. Structural parameters and exchange capacity of porous glass membranes. Colloid journal 1990. № 4. P. 774-777. (In Russ.)]

21. Филистеев О.В., Воронцов Б. С. Получение и свойства пористых стекол. Вестник КГУ 2011. № 2. C. 94-103. [Filisteev O. V., Vorontsov B. S. Obtaining and properties of porous glasses. Bulletin of $K G U$ 2011. № 2. P. 94-103. (In Russ.)]

22. Sato T., Funamori N., Yagi T. Helium penetrates into silica glass and reduces its compressibility. Nature Communications 2011. Vol. 2. P. 1-5.

23. Shen G., Mei Q., Prakapenka V.B., Lazor P., Sinogeikin S., Meng Y., Park C. Effect of helium on structure and compression behavior of $\mathrm{SiO}_{2}$ glass. PNAS 2011. Vol. 108 (15). P. 60046007.

24. Kuhtetskiy S.V., Fomenko E.V., Anshits A.G. Molecular dynamics simulation of helium diffusion in vitreous silica. Journal of Non-Crystalline Solids 2016. Vol. 443. P. 47-53.

25. Kukhtetskiy S.V., Fomenko E.V., Rogovenko E.S., Anshits A.G. Ab initio parameterization and testing of He and Ne effective potentials in silica. Journal of Non-Crystalline Solids 2020. Vol. 546. P. 120282. 